Revue internationale P.M.E.

Économie et gestion de la petite et moyenne entreprise

\title{
Crises et restructurations dans un système industriel localisé : l'exemple du textile à Roanne
}

\section{Françoise Rérat}

Volume 4, numéro 2, 1991

URI : https://id.erudit.org/iderudit/1008064ar

DOI : https://doi.org/10.7202/1008064ar

Aller au sommaire du numéro

\section{Éditeur(s)}

Presses de l’Université du Québec

\section{ISSN}

0776-5436 (imprimé)

1918-9699 (numérique)

Découvrir la revue

Citer cet article

Rérat, F. (1991). Crises et restructurations dans un système industriel localisé l'exemple du textile à Roanne. Revue internationale P.M.E., 4(2), 85-111. https://doi.org/10.7202/1008064ar

\section{Résumé de l'article}

Avec la crise économique, la dynamique de développement industriel émanant de la grande entreprise travaillant sur anticipations longues, avec des équipements rigides et une organisation taylorienne du travail, a été radicalement remise en cause. Des régions entières, organisées autour des grandes entreprises, ont été particulièrement touchées par la récession et le chômage. Dans le même temps, des régions composées majoritairement de PME non seulement se maintiennent dans la crise, mais ont parfois atteint un développement économique notable. Cette évolution favorable des PME dans la crise signifie-t-elle que les systèmes industriels locaux sont à l'origine d'un nouveau mode de développement économique ? Pour répondre à une telle question, il nous paraît nécessaire de retracer les grandes lignes de développement d'un système industriel localisé. Cette analyse sera réalisée à partir des événements qui ont affecté ce système dans le passé, et en particulier des restructurations qui l'ont suivi.

L'objet de cet article est d'analyser l'évolution de l'industrie textile dans la région de Roanne (ville industrielle française, proche de Lyon) à la fin des années 1980, en vue de rapporter l'émergence de nouveaux modèles de développement. La dispersion géographique et la petite taille des firmes qui, au milieu des années 60, apparaissaient comme un handicap face aux formes de modernisation imposées par la production en grandes séries, sont actuellement considérées comme des éléments de flexibilité et des facteurs de succès économique.

Les principaux arguments de l'étude concernent les facteurs de développement propres aux systèmes industriels localisés.

L'organisation de ia production nous apparaît comme le premier facteur de développement des systèmes industriels locaux. Les stratégies commerciales des firmes, en particulier, nous paraissent un facteur essentiel pour expliquer l'organisation d'un système productif local. Un troisième facteur d'analyse concerne le rôle des politiques de soutien au développement industriel local.

Notre analyse du système textile est basée sur des entretiens avec des chefs d'entreprises, des responsables locaux administratifs et politiques.

Nous avons relevé les modèles d'organisation de la production qui caractérisent le système industriel roannais avant la restructuration, pendant la crise, et après la restructuration. La première période, de 1964 à 1980, correspond à ce que nous avons appelé le " modèle de production traditionnel »; le début des années 1980 voit l'éclatement du modèle traditionnel et l'émergence de nouvelles tendances issues des nouvelles stratégies commerciales et des changements dans la qualité des produits; la fin des années 1980 consacre la stabilisation de ces nouvelles dynamiques de développement industriel.

Il reste à savoir si le système textile roannais, dans l'hypothèse où l'organisation actuelle se stabilise, constitue encore un système industriel local alors qu'il n'a plus de développement endogène et qu'il ne repose plus sur un modèle unique de développement
Ce document est protégé par la loi sur le droit d'auteur. L’utilisation des services d'Érudit (y compris la reproduction) est assujettie à sa politique d'utilisation que vous pouvez consulter en ligne.

https://apropos.erudit.org/fr/usagers/politique-dutilisation/ 


\title{
Crises et restructurations dans un système industriel localisé : l'exemple du textile à Roanne
}

\author{
Françoise RÉRAT * \\ Centre d'étude de l'emploi \\ FRANCE
}

\begin{abstract}
RÉSUMÉ
Avec la crise économique, la dynamique de développement industriel émanant de la grande entreprise travaillant sur anticipations longues, avec des équipements rigides et une organisation taylorienne du travail, a été radicalement remise en cause. Des régions entières, organisées autour des grandes entreprises, ont été particulièrement touchées par la récession et le chômage. Dans le même temps, des régions composées majoritairement de PME non seulement se maintiennent dans la crise, mais ont parfois atteint un développement économique notable. Cette évolution favorable des PME dans la crise signifie-t-elle que les systèmes industriels locaux sont à l'origine d'un nouveau mode de développement économique? Pour répondre à une telle question, il nous paraît nécessaire de retracer les grandes lignes de développement d'un système industriel localisé. Cette analyse sera réalisée à partir des événements qui ont affecté ce système dans le passé, et en particulier des restructurations qui l'ont suivi.
\end{abstract}

L'objet de cet article est d'analyser l'évolution de l'industrie textile dans la région de Roanne (ville industrielle française, proche de Lyon) à la fin des années 1980, en vue de rapporter l'émergence de nouveaux modèles de développement. La dispersion géographique et la petite taille des firmes qui, au milieu des années 60, apparaissaient comme un handicap face aux formes de modernisation imposées par la production en grandes séries, sont actuellement considérées comme des éléments de flexibilité et des facteurs de succès économique.

Les principaux arguments de l'étude concernent les facteurs de développement propres aux systèmes industriels localisés.

* Françoise Rérat née à Lyon le 3 octobre 1931. Études de psychologie et d'économie (DEA). Psychologue chargée de la sélection de cadres techniques et commerciaux dans un cabinet de consultants pendant 5 ans. Chargée de recherche au Centre d'Études de l'Emploi spécialisée sur la socio-économie des petites et moyennes entreprises industrielles. Adresse : Centre d'études de l'emploi, « Descartes I », 29 Promenade Michel Simon, 93191 - Noisy Le Grand, Cedex France. 
L'organisation de la production nous apparaît comme le premier facteur de développement des systèmes industriels locaux. Les stratégies commerciales des firmes, en particulier, nous paraissent un facteur essentiel pour expliquer l'organisation d'un système productif local. Un troisième facteur d'analyse concerne le rôle des politiques de soutien au développement industriel local.

Notre analyse du système textile est basée sur des entretiens avec des chefs d'entreprises, des responsables locaux administratifs et politiques.

Nous avons relevé les modèles d'organisation de la production qui caractérisent le système industriel roannais avant la restructuration, pendant la crise, et après la restructuration. La première période, de 1964 à 1980, correspond à ce que nous avons appelé le "modèle de production traditionnel "; le début des années 1980 voit l'éclatement du modèle traditionnel et l'émergence de nouvelles tendances issues des nouvelles stratégies commerciales et des changements dans la qualité des produits; la fin des années 1980 consacre la stabilisation de ces nouvelles dynamiques de développement industriel.

II reste à savoir si le système textile roannais, dans l'hypothèse où l'organisation actuelle se stabilise, constitue encore un système industriel local alors qu'il n'a plus de développement endogène et qu'il ne repose plus sur un modèle unique de développement.

\section{ABSTRACT}

The economic crisis has brought into question the dynamics of industrial development based on the model of the large firm. Entire regions organized around this model have been affected by the economic recession and by unemployment, whereas other regions having a majority of small and medium sized firms have not only survived the crisis but even managed to reach a substantial rate of economic growth. Even if they are currently successful, however, will local industrial systems be able to face the reorganization of the international market? In order to answer this question one must outline the patterns of development of local industrial systems. We will do this through an analysis of the events which have affected these systems and particularly of the restructurations which follow these events.

The purpose of this essay is to analyze the trends in the textile industry in Roanne (an industrial town near Lyon in France) at the end of the 1980's, in order to identify its emerging patterns of development. The geographical dispersion and the small size of the firms, which were seen, in the middle of the 1960 's, as shortcomings in terms of the forms of modernization imposed by long-run production, are now considered as elements of flexibility and factors of economic success.

The main arguments of the study address the factors of development of local small and middle-sized firms.

To start with, we consider the organization of production as the main factor accounting for the development of local industrial systems. This becomes clear when product quality is taken into account - a factor these analyses neglect. $A$ 
third factor is the impact of public policies on the development of local industrial systems. The importance of this factor has been correctly pointed out by analyses in terms of "flexible specialization" or "scattered industrial organization".

Our analysis of the textile industrial system in Roanne is based on interviews with managers and local business and political leaders.

We have identified the models of the organization of production which have characterized the industrial system in Roanne before the restructuring, during the crisis, and after the restructuring. The firts period, 1964-1980, correspond to what we have named the traditional model of production; the beginning of the 1980 's corresponds to a departure from that traditional model and to the emergence of new industrial patterns linked to commercial strategies and to changes in product quality; finally, the end of the 1980's saw the stabilization of these new industrial patterns.

Thus, any hypothesis about the stability of the current organization of the Roanne industrial system needs to be put into historical perspective, which leads one to wonder if the textile system in Roanne still constitutes a local industrial system, since, even if there are successful strategies, there no longer is an endogenous or unique model of development.

\section{RESUMEN}

La crisis económica ha puesto en cuestión la dinámica del desarrollo industrial basada en el modelo de la gran empresa. Regiones enteras organizadas alrededor de este modelo se han visto afectadas por la recesión económica y el desempleo, mientras que otras regiones que cuentan con una mayoría de pequenas y medianas empresas no sólo sobrevivieron a la crisis sino que han logrado obtener un importante crecimiento de la tasa económica. Sin embargo; podran hacer frente a la reorganizacion del mercado internacional los sistemas industriales locales, aun si actualmente tienen éxito? Para responder a esta pregunta hay que tomar en cuenta las características del desarrollo en los sistemas industriales locales. Haremos esto a través de un análisis de los hechos que han afectado estos sistemas y particularmente sobre las restructuraciones suscitadas por aquellos acontecimientos.

A fin de identificar las nuevas características del desarrollo, este ensayo se propone analizar las tendencias de la industria textil en Roanne (ciudad industrial ubicada cerca de Lyon, Francia), a fines de los años ochenta. Si bien a mediados de los años sesenta la dispersión geográfica y la pequena envergadura de las empresas eran consideradas ineficientes desde el punto de vista de las formas de modernizacion impuestas por la producción a largo plazo, hoy en día se las considera como elementos de flexibilidad y factores de éxito económico.

Los argumentos mas importantes del estudio se refieren a los factores de desarrollo en las pequenas y medianas empresas locales. 
En primer lugar consideramos que el factor principal responsable del desarrollo de los sistemas industriales locales es la organización de la producción. En particular, se puede decir que las estrategias comerciales de las empresas afectan en gran medida la organización de los sistemas industriales de producción a nivel local. Un tercer factor es el impacto de las políticas gubernamentales en cuanto al desarrollo de sistemas industriales locales.

Nuestro análisis del sistema industrial textil en Roanne se basa en entrevistas con directores, empresarios locales y lideres politicos. Hemos identificado los modelos para la organización de la producción que han caracterizado el sistema industrial en Roanne antes de la restructuración, durante la crisis, y despues de la restructuración. El primer periodo, 1964-1980, corresponde a lo que hemos calificado como el modelo tradicional de producción; los primeros años de los ochenta corresponden de nuevas pautas industriales asociadas a estrategias comerciales y cambios en la calidad del producto; por último, a fines de los años ochenta se llega a la estabilización de las nuevas pautas industriales.

Asimismo, toda hipótesis sobre la estabilidad de la organizacion actual del sistema industrial en Roanne necesita ser visto desde una perspectiva histórica. Podriamos decir que el sistema textil en Roanne sigue siendo un sistema industrial local pues, si bien tiene estrategias de éxito, ya no existe un modelo de desarrollo único o endogeno.

\section{Introduction}

Avec la crise économique, la dynamique de développement industriel émanant de la grande entreprise travaillant sur anticipations longues, avec des équipements rigides et une organisation taylorienne du travail, a été radicalement remise en cause. La recherche d'une meilleure adaptation aux nouvelles donnes du marché a entraîné l'abandon progressif des structures anciennes en vue d'aboutir à plus de souplesse et de flexibilité dans l'organisation de la production. On assiste à une délocalisation d'une partie des activités de production vers des pays à bas coûts de main-d'œuvre ainsi qu'à leur recomposition dans des petites unités à effectifs réduits.

La restructuration des grandes entreprises a entrainé des ruptures graves dans l'équilibre socio-économique local: les régions organisées autour des grandes entreprises ont été particulièrement touchées par la récession et le chômage. Dans le même temps, des systèmes de PME locaux, non seulement se maintiennent dans la crise mais atteignent un développement économique notable. Cette situation privilégiée des PME dans la crise a poussé les chercheurs à s'interroger plus précisément sur le rôle des PME en tant que moteur possible d'une nouvelle forme de développement économique. 
En fait, cette réussite des PME n'est pas nouvelle dans l'histoire économique des pays avancés : tout au long du XIX $x^{e}$ siècle et durant la première moitié du $\mathrm{xx}^{\mathrm{e}}$ de nombreuses régions se sont développées grâce à ces systèmes souples de production organisés autour d'une activité dominante comme la soierie dans la région lyonnaise ou la coutellerie à Sheffield. Mais avec la prédominance de la grande entreprise dans l'économie de la plupart des pays avancés, ces systèmes de PME semblaient voués à disparaître. L'exemple de la soierie lyonnaise est très illustratif à cet égard. Ce système, qui avait surmonté victorieusement plusieurs crises jusqu'en 1960, s'est effondré à la suite de la restructuration suscité par la politique publique d'aide à la constitution de groupes à dimension internationale (Piore et Sabel, 1984). Les réseaux très souples de spécialistes dont la région s'était dotée ont disparu et les différents corps de métier ont été intégrés dans de nouvelles organisations (Rhône-Poulenc, DMC...) qui poursuivaient des stratégies de production en séries pour le marché mondial. Dans le même temps, toute la production spécialisée s'est repliée sur la région de Côme (Laférère, 1960).

Tandis que des systèmes de PME disparaissaient de certaines régions, d'autres ont surgi et se sont développés de manière spectaculaire, en particulier en Italie, provoquant ce qu'il est convenu d'appeler « le miracle italien ». La réussite du modèle italien de « district industriel » depuis la fin des années 1960 a focalisé l'intérêt des économistes et sociologues italiens bien avant que les chercheurs des autres pays ne s'intéressent à ce mode d'organisation productive. Plusieurs concepts ont été élaborés pour caractériser ce phénomène : décentralisation productive (Brusco, 1980) développement périphérique (Bagnasco, 1977), district industriel, aire système (Beccatini, 1987) etc.

Dans la recherche que nous avons menée sur le système textile roannais, la référence au concept de «district industriel » est constante. Toutefois, nous lui préférons le terme de "système industriel localisé " (Roveyre et Saglio, 1984), le district industriel étant, selon nous, spécifique à l'organisation productive des systèmes de PME italiens.

Sans nous engager dans une analyse approfondie de ce concept, lequel déborderait le cadre d'un article consacré à une recherche sur le terrain, il nous semble indispensable de rappeler tout d'abord les principales caractéristiques du district industriel telles qu'elles ont été définies récemment (Beccatini, 1989). Nous situerons ensuite notre propre démarche par rapport à ce concept et nous verrons en quoi il constitue un cadre d'analyse des stratégies de développement industriel à l'œuvre dans la région roannaise. En tant que modèle de développement endogène, ce concept nous semble parfaitement explicatif d'un stade de développement industriel historiquement daté, que nous avons dénommé « modèle de production traditionnel » lequel caractérise le mode de développement en cours pendant les années 1960. Mais est-il en mesure de rendre compte des 
transformations actuelles de l'organisation productive? D'autres variables explicatives nous semblent indispensables à prendre en compte pour mesurer ces transformations, en particulier le rôle des politiques menées par les firmes en matière de qualité des produits et le rôle des crises qui affectent l'organisation globale d'un système industriel localisé. Dans la deuxième partie de cet article, nous présenterons notre analyse du système textile roannais en la situant par rapport aux deux dernières crises qui ont touché la région.

\section{La place du district dans notre analyse du système textile roannais}

\subsection{Définition du district industriel}

Beccatini (1989) distingue des caractéristiques économiques, et d'autres relevant du fonctionnement social. Le processus de production est éclaté en une multitude de phases autonomes correspondant à des unités indépendantes spécialisées. Le district vit en symbiose avec l'extérieur et non en autarcie ; les entreprises sont en concurrence avec les firmes extérieures, tout en ayant des échanges très poussés à l'intérieur du district. Le mode de développement industriel est généralement endogène, bien qu'il puisse être impulsé dans certains cas par des interventions extérieures ( $c f$. l'aide fournie par les collectivités territoriales au démarrage industriel de l'Emilie Romagne après la Deuxième Guerre mondiale). L'organisation de la production est extrêmement flexible grâce au réseau de sous-traitance. En ce qui concerne les ressources humaines, on relève trois points principaux : la mobilité des personnes qui s'appuie sur un continuum social autorisant les projets d'ascension les plus larges depuis le bas de l'échelle jusqu'au statut prestigieux d'entrepreneur local, la professionnalité (enracinement local des chefs d'entreprise), la flexibilité sociale liée à un réservoir de maind'œuvre féminine mobilisable en fonction des fluctuations de la demande des entreprises.

L'apport majeur de ce concept de district industriel, selon nous, a été de focaliser l'intérêt des chercheurs sur des variables généralement séparées dans l'analyse courante parce que renvoyant à des disciplines différentes - économie, sociologie, géographie industrielle. L'analyse porte tout autant sur la nature et la qualité des investissements productifs que sur les stratégies développées par les acteurs locaux, le rôle de la famille élargie. Nous rejoignons ce courant de recherche sur un point précis qui est celui de la convergence établie entre l'organisation de la production et l'organisation du travail. En nous appuyant sur deux analyses qui nous ont servi de référence dans notre recherche, nous allons maintenant présenter notre propre démarche d'analyse du système textile roannais. 


\subsection{L'organisation de la production}

Les systèmes industriels locaux sont-ils la survivance d'une organisation de la production qui semblait en voie d'extinction parce que relevant du modele artisanal ? Et dans ce cas, comment ont-ils survécu en proposant, certes, des biens attrayants parce que répondant à des besoins spécifiques, mais trop chers comparés aux produits fabriqués en grandes séries et bénéficiant d'économies d'échelles? Selon Piore et Sabel, il s'agirait d'une organisation artisanale renouvelée, employant des techniques sophistiquées, mais douées d'une grande souplesse de gestion et d'une stratégie d'innovation permanente. On peut parler, selon ces auteurs, d'une renaissance des formes de production artisanales marginalisées lors du premier clivage industriel qui s'est opéré à la fin du siècle dernier à l'avantage de la grande entreprise. C'est ce qu'ils ont défini comme le modèle de « spécialisation flexible" (Piore et Sabel, 1984).

D'autres auteurs (Brusco, 1982) considèrent que ces systèmes localisés sont issus de la « désintégration verticale de la production " générée par l'éclatement de la grande entreprise intégrée. Dans les districts italiens, il ne s'agirait plus maintenant d'une organisation artisanale, mais d'une organisation industrielle éclatée en une multitude de phases productives autonomes. Au modèle de « l'artisan » aurait succédé à la fin des années 1960, le modèle du « sous-traitant dépendant » puis celui du « district industriel » proprement dit.

L'accent mis sur le primat de l'organisation de la production dans ces deux analyses paraît essentiel pour bien comprendre le fonctionnement des systèmes productifs locaux. Qu'il s'agisse d'organisation " artisanale renouvelée " ou d'organisation " industrielle éclatée ", c'est la flexibilité d'ensemble du système qui est à l'origine de la réussite. Sous le terme d'organisation flexible de la production, ces auteurs mettent en relation deux niveaux d'analyse : l'organisation au niveau de l'atelier, qui passe par des méthodes de gestion souple de la force de travail (mobilité, travail temporaire, travail à domicile, etc.) et l'organisation globale de la production d'un système d'entreprises qui désigne les différentes stratégies industrielles à l'œuvre localement. Mais y a-t-il réellement homogénéité entre les deux niveaux ? Un système de production flexible est-il entièrement composé de segments flexibles? N'y a-t-il pas certains segments très taylorisés, et d'autres où le savoir-faire et la polyvalence sont utilisés ?

Dans le premier stade défini par Brusco, celui de «l'artisan », la concurrence s'établit entre des producteurs égaux, et c'est le savoir-faire de l'artisan qui déclenche la réussite de l'entreprise. Au deuxième stade, celui du "soustraitant dépendant » apparu au cours des années 1960, s'instaure un rapport de domination entre donneur d'ordre et sous-traitant qui infere une organisation du travail différente dans les deux types d'entreprises. Au troisième stade, se développe un système de solidarités réciproques entre tous les producteurs. Mais ce 
système fait-il disparaître les clivages antérieurs ? Va-t-on vers une unification de l'organisation du travail?

Cette analyse globale de l'ensemble des entreprises du district ne prend pas en compte la diversité des stratégies industrielles à l'intérieur du district et pousse à réduire l'organisation productive à un seul modèle de développement, celui de la spécialisation flexible. On imagine un scénario unique en ce qui concerne les conditions de travail : la flexibilité s'associe à la polyvalence pour éliminer progressivement les segments taylorisés utilisateurs de main-d'œuvre nombreuse et non qualifiée. N'y aurait-il pas d'autres évolutions possibles ?

L'analyse que nous avons menée à Roanne nous amène à penser qu'il existe des stratégies diverses à l'intérieur d'un système de PME localisé qui combinent la production en grandes séries et la spécialisation flexible, et qu'il y a plus d'un modèle d'organisation productive. C'est ce qui se dessine très nettement dans cette région après la période de restructuration qui a vu l'effondrement d'une organisation productive très homogène centrée sur un seul modèle de réussite, celle d'un grand fabricant roannais de prêt-à-porter féminin. De nouvelles stratégies industrielles assez différenciées sont apparues, aboutissant à un remodelage des relations à l'intérieur de la zone. Plusieurs modèles d'organisation se sont mis en place, combinant de manière diverse les investissements techniques et les investissements commerciaux, la flexibilité du travail et la polyvalence, les produits à renouvellement rapide et les produits plus traditionnels, les petites séries et les grandes.

Pour cerner la diversité des stratégies industrielles à l'œuvre dans cette région, d'autres variables ont été prises en compte, en particulier les politiques menées par les firmes en matière de qualité des produits.

\subsection{Organisation flexible et qualité des produits}

Le choix d'une production souple en petites séries diversifiées, qui caractérise les systèmes industriels locaux, vise à réaliser des produits compétitifs adaptés à l'évolution de la demande. Les entreprises abandonnent la recherche d'économies d'échelles pour privilégier la qualité du produit. Cette recherche d'une meilleure compétitivité s'accompagne souvent d'un changement dans la qualité des produits. Elle se manifeste à l'intérieur de l'entreprise à travers ce qu'on a coutume d'appeler les cercles de qualités et à l'extérieur de l'entreprise dans les liens privilégiés et durables qui sont noués avec le sous-traitant et avec les clients. François Eymard-Duvernay et Olivier Favereau (1990), se référant à la théorie des marchés internes de Dœringger et Piore, établissent une relation entre « la qualité des produits et la qualité des emplois ». Selon ces auteurs, la qualité des produits découle des politiques de gestion des entreprises. En d'autres termes, la qualité des produits varie en fonction des différents modes 
d'organisation de la production et de gestion de la force de travail : à un mode de production «domestique" correspond un produit dont la qualité dépend étroitement d'un savoir-faire traditionnel et d'une gestion familiale de la maind'œuvre ; à un mode de production « industriel » correspond un produit dont la qualité dépend des investissements techniques de l'entreprise et de ses capacités d'innovation (Eymard-Duvernay, 1990).

Ce concept nous paraît intéressant à appliquer au cas des systèmes de PME locaux. En effet, ces systèmes constituent des entités économiques aisément mesurables du fait de leur localisation géographique et de leur durabilité dans le temps. Ils permettent de situer l'évolution de la qualité par rapport à l'évolution d'un système d'entreprises. Un système local est composé d'entreprises dont la diversité concourt à la réalisation de produits variés. La qualité des produits réfère tout autant aux donneurs d'ordre fabriquant des produits de marque qu'aux petites entreprises sous-traitantes. Pour une entreprise donnée, la qualité des produits dépend aussi bien de son organisation propre que de son insertion dans le système local (existence d'une main-d'œuvre qualifiée, possibilités de travail à domicile). Par ailleurs, la qualité du produit varie dans le temps, pas seulement en fonction des aléas conjoncturels du marché. Elle varie aussi en fonction des crises qui affectent le système et des restructurations qui les suivent.

En reprenant l'exemple de la soierie lyonnaise cité précédemment, on voit en effet comment la qualité du produit a évolué à travers les crises successives qui ont touché l'organisation de ce système de production.

Au départ, dans la première moitié du $\mathrm{xIX}^{e}$ siècle, il s'agissait d'un système entièrement intégré (comparable à la grande entreprise éclatée de Brusco), depuis la fabrication de la matière première dans les régions avoisinantes (élevage des vers à soie) jusqu'au tissage dans les petits ateliers lyonnais. Le critère de la qualité du produit dépendait de la qualité de la matière première utilisée : la soie, matière noble par excellence. Le poids et l'épaisseur du tissu étaient le garant de la qualité. La soie était un produit de qualité stable, invariable dans le temps (soies noires lourdes et empesées, style Reine Victoria) (Lafèrère, 1960).

Après l'épidémie qui ravagea les élevages de vers à soie et les fit disparaître, les négociants lyonnais se sont mis à importer la soie en provenance d'Extrême Orient. Les tissus sont devenus plus légers, à cause de l'élévation du prix de la matière première. Le critère de la qualité du produit a été celui de la légèreté et de la souplesse : à ces qualités se sont ajoutés les coloris multiples grâce aux recherches et aux innovations dans la teinture. La soierie est devenue un produit riche et diversifié en même temps qu'un produit mode de haute nouveauté. 
La troisième époque, qui correspond à la crise des années 1930 , a vu apparaître ce qu'on pourrait définir comme une qualité industrielle : des fibres artificielles sont mélangées aux fils de soie et c'est la parfaite imitation du tissu de soie qui devient le nouveau critère de la qualité du produit. Aux avantages financiers reliés au changement de matière première, s'ajoutent les qualités propres au nouveau produit ${ }^{1}$ qui entraînent un développement considérable de la demande. Le savoir-faire traditionnel reste encore dans les opérations de tissage et de teinture, malgré l'évolution du produit vers une qualité plus industrielle.

À partir des années 1960, s'amorce la rupture entraînée par la restructuration : la qualité industrielle est devenue l'apanage des grands groupes de la chimie qui disposaient de leur propre matière première et de moyens techniques pour assurer cette qualité (tissus entièrement en fibres synthétiques pour une consommation de masse), alors que la qualité traditionnelle s'est trouvée limitée à quelques survivants de l'ancien système, situés dans la région roannaise et le haut Beaujolais, spécialisés dans les tissus de soie pour la haute couture.

Dans les systèmes industriels locaux qui se maintiennent et se développent aujourd'hui, la souplesse de l'organisation de la production vise à définir de nouvelles normes de qualité qui échappent au dilemme qualité industrielle production de masse, qualité artisanale - C. production de luxe. Dans ces systèmes flexibles, la qualité du produit signifie : réduction des délais de production, réactivité, créativité dans les formes plus que dans la matière première, copie des produits de luxe, renouvellement des couleurs. Désormais, la qualité des produits dépend des nouvelles stratégies commerciales qui elles-mêmes déterminent l'organisation de la production.

Ces stratégies commerciales ne sont pas seulement le fait des entreprises productrices. Les grands groupes de distribution ont eux aussi des stratégies commerciales qui visent le consommateur final. Leur position dominante sur certains marchés, du fait qu'ils importent une grande partie des produits qu'ils écoulent, a des répercussions importantes sur l'organisation des systèmes locaux et sur l'évolution de la qualité des produits. Constituant un écran entre les producteurs et le marché final, la politique des grands distributeurs aboutit à une nouvelle segmentation des produits, poussant certains producteurs à se repositionner sur des marchés que la grande distribution n'atteint pas, et d'autres à devenir des sous-traitants travaillant pour la grande distribution. On voit ainsi se dessiner de nouveaux systèmes de production dans lesquels la qualité des produits se différencie en fonction de la capacité de résistance et d'adaptation de ces systèmes à la domination des grands groupes de distribution.

1. Facile à laver, indéfroissable. 
Cette situation illustre le cas de certains systèmes locaux en France, spécialisés dans le textile et la chaussure. En Italie, dans les districts de la chaussure étudiés récemment, la situation est très différente du fait que l'industrie de la chaussure est fortement exportatrice et qu'il n'y a pas de concentration au niveau de la distribution, comme c'est le cas en France et en Grande-Bretagne (Courault et Rérat, 1990). Les acheteurs étrangers se déplacent dans les régions productrices et passent leur commande directement aux entreprises. Les producteurs gardent la maîtrise de la qualité du produit. Cette dernière est liée à la très grande créativité et à la capacité des entreprises de copier les produits en vogue. Par contre, les rythmes de production restent ceux d'une industrie saisonnière organisée autour des collections.

\subsection{Les crises qui affectent les systèmes industriels locaux et le rôle des politiques de soutien au développement industriel}

L'analyse des districts industriels en Italie a été réalisée pendant une période de forte croissance. La réussite de ces systèmes était alors incontestable ${ }^{2}$. Mais l'évolution récente de l'économie italienne semble remettre en cause ce tableau idyllique. La crise que subit depuis quelques années la région de Prato laisse apparaître que les systèmes souples de production ne sont pas à l'abri des crises suscitées par l'accélération de la concurrence internationale. Néanmoins, ces systèmes industriels persistent, même s'ils subissent des mutations. Il nous paraît important d'étudier l'histoire des systèmes locaux à travers les crises qui les affectent et de voir comment s'opèrent les restructurations qui suivent ces crises. C'est dans ce domaine que les comparaisons internationales peuvent être fructueuses. Quand on suit l'évolution dans le temps d'un système de PME localisé, on peut en effet mesurer sa capacité de résistance et d'adaptation aux crises qui l'assaillent. La baisse des effectifs employés n'est pas toujours un signe de déclin.

On s'extasie actuellement sur la capacité de résistance à la crise des systèmes de PME locaux, mais il ne faut pas oublier leur fragilité liée à la petite taille des entreprises et au fait que la réussite de l'entreprise repose sur les qualités de la personne qui la dirige. Par ailleurs, il s'agit pour la plupart d'entreprises familiales: des problèmes peuvent surgir lors des successions et se répercuter sur l'ensemble de la région. C'est là qu'interviennent certaines associations locales d'assistance juridique, technique et commerciale qui offrent leurs services aux entreprises de la région.

En France, les systèmes de PME localisés se sont développés généralement sans aide des collectivités territoriales. Leur situation géographique en zone rurale, hors des grands axes routiers et ferroviaires a favorisé la constitution

2. Leur avenir, assuré. 
d'un système de relations et de solidarités relativement fermé au monde extérieur. Certains systèmes industriels locaux se sont créés en mobilisant l'épargne locale (Minguet, 1985). Depuis la crise, cette situation a évolué et des politiques d'aide de l'État aux entreprises ont été développées. On peut citer, à titre d'exemple, le Plan textile 1982-1983 qui a incité les entreprises à moderniser leurs équipements. Des politiques locales passant par le biais des Chambres de Commerce locales, des associations patronales, visent à ouvrir les régions sur le monde extérieur, à développer l'innovation, à faire circuler les informations. De nouvelles synergies se manifestent. Et là encore, des différences apparaissent, certains systèmes continuent à fonctionner, d'autres s'écroulent. Et se pose la question des limites des politiques locales qui ne peuvent maintenir en vie des systèmes qui n'ont pas de vie propre. On touche là des facteurs qui semblent déterminants bien que difficiles à mesurer, tels que la mentalité entrepreneuriale, la capacité d'adaptation des salariés aux changements de cap de l'entreprise, l'attachement au pays.

\section{L'analyse du système roannais}

Cette étude a été réalisée en période de postrestructuration à la suite d'une crise qui a fortement ébranlé la région et classé Roanne parmi les zones sinistrées. Le système textile roannais est sorti affaibli par cette crise en ce qui concerne le nombre d'entreprises et les effectifs employés ${ }^{3}$. Mais il a retrouvé actuellement un nouveau dynamisme, ce qui n'est pas le cas d'autres régions textiles, tel Troyes. Parmi les hypothèses qu'on peut formuler pour expliquer la capacité de résistance et de renouvellement du système textile roannais, on en retiendra deux principales. La première hypothèse tient à la souplesse de structure du système roannais composé d'une multitude de PME familiales. La deuxième renvoie à la politique d'intervention de l'État. À travers le Plan textile 81/82 l'aide aux entreprises n'a pas visé une restructuration du tissu industriel sous l'égide de grandes entreprises appartenant à des groupes (comme ce fut le cas dans les années 60), mais une aide technique aux PME favorisant les investissements de modernisation.

L'enquête que nous avons réalisée se situe à une époque qui est le point d'aboutissement de plus d'un siècle et demi d'industrialisation. Le bref aperçu historique qui va suivre permettra de mieux situer l'évolution du système textile roannais dans le temps. On a tenté de dégager les modèles d'organisation de la production correspondant à deux époques: la fin des années 1960 jusqu'au

3. Entre 1975 et 1985 , les effectifs employés dans le textile ont baissé de $38 \%$. $C f$. Lisiane Cartellier et Bernard Simonin, « Formation continue et développement des PME », Rapport CREDOC pour le Plan, janvier 1990. 
début des années 1980 correspond à ce que nous avons appelé le modele de production traditionnelle; la fin des années 1980 voit l'éclatement du modèle traditionnel et l'émergence de nouvelles dynamiques industrielles liées aux stratégies commerciales et aux changements dans la qualité des produits.

\subsection{Origines et développement du système textile roannais jusqu'à la crise du tissage en 1964}

Bien que relativement proche de Lyon $(90 \mathrm{~km})$ et de son industrie de la soie, le système textile roannais s'est développé de manière autonome depuis un siècle et demi, du fait de sa situation géographique enclavée, au milieu de montagnes, loin des grands axes routiers et ferroviaires. Composé d'une multitude de petites et moyennes entreprises dans lesquelles a longtemps persisté la complémentarité travail agricole/travail à domicile des femmes, ce système regroupe encore actuellement tous les maillons de la filière textile-habillement, excepté la filature ${ }^{4}$. Bien que le secteur des industries métallurgiques et mécaniques soit relativement important, il ne s'est pas développé autour du textile : il n’y a pas d'activité de construction de machines textile ${ }^{5}$. Le tissage, resté longtemps l'activité dominante, est relayé depuis le milieu des années 1960 par la mailleconfection qui est devenue la première industrie textile de Roanne.

La tradition du tissage est très ancienne dans la région roannaise, comme dans beaucoup de régions rurales. Pendant des siècles, les métiers à tisser ont voisiné avec les outils servant aux travaux des champs. Au tissage du coton et du chanvre, s'est petit à petit adjoint à partir du XvIr eiècle le tissage de la soie : les artisans des montagnes roannaises travaillaient pour le compte des grandes maisons de soieries lyonnaises. La tradition de la bonneterie remonte au Second Empire. À cette époque, l'activité artisanale des femmes de la région qui tricotaient à la main, au crochet ou à l'aiguille, a commencé à s'organiser sur le plan commercial. Des "fabriques de lainage" sont apparues qui vendaient des pèlerines, châles, capulets et layettes, exclusivement faits à la main par des ouvrières à domicile, en ville ou aux champs ${ }^{6}$. C'est vers 1880-1890 qu'une véritable industrie textile régionale est apparue, avec les premiers métiers mécaniques, aussi bien en tissage qu'en tricot ${ }^{7}$.

4. L'industrie textile roannaise regroupe en 1989 près de 10500 salariés. Elle représente $42 \%$ des activités industrielles et la métallurgie-mécanique, $26 \%$.

5. La seule entreprise fabriquant du matériel textile a disparu au début des années 1980.

6. Le tricot et la broderie à la main ont perduré dans la région jusqu'à la fin des années 1960.

7. Venus d'Allemagne, les premiers métiers à tricoter étaient encore basés sur le principe des métiers à tisser. Ils utilisaient un rouleau de chaîne, mais la navette qui constitue la trame était réalisée par une série d'aiguilles à tricoter qui formaient une « maille ». $C f$. Dépêche Mode, juillet-août 1975. 
Le système textile roannais ne s'est pas développé autour de grandes entreprises situées en milieu urbain comme ce fut le cas dans les grandes villes, mais par une dispersion des petites unités de tissage dans les bourgs avoisinant Roanne. Cette dispersion a été rendue possible grâce à l'avènement de l'électricité. De plus, selon Jean-Pierre Houssel (1978), elle répondait à la crainte de la bourgeoisie locale à l'égard de la montée du socialisme dans les grandes villes. «[...] La bourgeoisie issue des classes moyennes de la montagne tourne le dos à la concentration urbaine et au progrès technique. Elle épouse l'idéologie de l'aristocratie qui prône la supériorité de la campagne et de la vie traditionnelle " (Houssel, 1978). Ce dernier trait est particulièrement significatif d'une certaine mentalité entrepreneuriale qui règne encore aujourd'hui dans la région.

L'industrie du tissage a eu une croissance continue à partir de la guerre de 14-18. Les usines deviennent très mécanisées avec l'introduction des métiers automatiques. Elles utilisent une main-d'œuvre nombreuse, peu qualifiée qui émigre des campagnes voisines vers les centres urbains de la région. L'entreprise de tissage constitue l'employeur majoritaire et assure la pérennité de l'emploi. On se retrouve de père en fils à travailler dans la même usine. Roanne devient un centre de tissage de coton réputé : la grande spécialité de la région est la toile de Vichy ${ }^{8}$. D'autres étoffes comme les cotons fins, teints en fils, sont employés en haute couture ou destinés à l'exportation. Chaque centre de la région est spécialisé dans un type de produit, linge de table à Panissières, couvertures à Cours, éponge à Thisy, voilages à Tartare. La matière première de référence est le coton. Mais apparaissent, à la fin des années 1950 de nouvelles fibres synthétiques auxquelles s'adjoignent des techniques modernisées de tissage qui viennent diversifier les produits roannais et élargissent la demande.

L'industrie de la bonneterie s'est développée de manière foudroyante après la guerre de 14-18, grâce à l'apparition successive des métiers « rectilignes » puis des métiers "circulaires ». Les firmes naissent comme des champignons. La crise de 1929 stoppe cette expansion ${ }^{9}$. Après la Deuxième Guerre mondiale, Roanne devient un centre de bonneterie spécialisé dans la confection pour femme, la layette et les vêtements d'enfants. L'implantation régionale est assez diversifiée, mais avec une certaine concentration dans la ville de Roanne. La matière première de référence est la laine. Mais comme pour le tissage, des fibres nouvelles sont tricotées qui permettent une diversification des produits.

8. Tissus à petits carreaux, très en vogue jusqu'aux années $1960, c f$. les robes de Brigitte Bardot dans ses premiers films.

9. Le nombre d'entreprises de bonneterie reste inchangé depuis cette période jusqu'aux années 1950, période d'une nouvelle expansion correspondant à l'arrivée dans ce secteur de familles israélites qui ont créé de nouvelles entreprises et apporté un changement. 
La prospérité du textile roannais s'est maintenue jusqu'au début des années 1960. À partir de 1963, une grave crise touche la région, provoquant l'effondrement du tissage, en tant qu'activité dominante ${ }^{10}$. L'organisation du système productif roannais n'était pas adaptée à l'évolution de la conjoncture internationale, c'est-à-dire à l'arrêt du protectionnisme par suite de l'ouverture des frontières. La dispersion et la petite taille des entreprises qui aujourd'hui sont considérées comme un élément positif d'adaptation à la crise, apparaissaient à l'époque comme un handicap face à la modernisation imposée par la production en grandes séries.

Comme la plupart des systèmes industriels locaux qui subsistaient en France à cette époque, le système textile roannais a été touché par le mouvement de modernisation lancé par les planificateurs, visant à faire sortir la France de son état «d'arriération industrielle » (Ganne, 1990) et à développer des structures productives organisées autour de grands groupes industriels représentant l'ensemble de la filière de production. La restructuration a touché le tissage avec l'absorption de six firmes roannaises par le groupe DMC ${ }^{11}$. D'autres groupes étaient déjà présents dans la teinture (Gilet Thaon, puis Chargeur) et dans le tissage de rayonne (Rhône Poulenc). La bonneterie n'a pas été touchée par la restructuration, malgré un léger fléchissement d'activité au moment de la crise. On a enregistré quelques fermetures d'entreprises, mais il n'y a pas eu d'absorptions par des groupes. Il semble que ce soit la structure même de cette activité composée de petites et moyennes entreprises autour desquelles gravitent une multitude de petits façonniers et d'artisans qui ait été à l'origine de sa réussite et de son maintien à l'écart des restructurations.

\subsection{Le modèle de production traditionnel}

Nous avons tenté de reconstituer le modèle de production industrielle qui a servi de référence à la bonneterie jusqu'à la fin des années 1970, période où une nouvelle crise a secoué la région et remis en cause l'organisation productive dans sa globalité. Ce modèle emprunte quelques traits à un grand fabricant de prêts-à-porter féminins spécialisé dans le haut de gamme qui a représenté, pendant la période de croissance, l'image de la réussite de la région ${ }^{12}$. Il décrit une dynamique de développement industriel qui plonge ses racines dans la tradition locale.

10. Entre 1963 et 1973 , plus de 3000 emplois ont disparus dans le tissage, et de nombreuses entreprises ont fermé leurs portes.

11. Dolfüs Mieg est un groupe textile important, originaire des Vosges, spécialisé dans la filature (Thiriez et Cartier-Bresson) et le tissage.

12. Cette entreprise représente par excellence le modèle «mythique» du fabricant roannais, tant par son importance, son image de marque, sa politique sociale paternaliste. À Roanne, quand on parle de cette entreprise, on parle du château. 
Dans ce système de production traditionnelle, le fabricant réalise à l'intérieur de l'entreprise toutes les opérations de fabrication depuis la conception du produit jusqu'à la réalisation du produit fini et confie à la sous-traitance les surplus d'activités reliés aux fluctuations saisonnières. Cette organisation pousse à une domination très forte des fabricants sur les façonniers qui sont dans l'incapacité de réguler leur production propre. Cela explique le développement d'un "système de production souple " accru encore par la très grande proximité des entreprises à l'intérieur de la ville de Roanne ${ }^{13}$. Ce système fonctionne avec des échanges de services dans l'environnement immédiat, les façonniers étant à portée de la main pour répondre aux à-coups de production. Ces entreprises installées dans des locaux exigus, mal adaptés à la production industrielle ont toujours eu recours de façon considérable au travail à domicile, afin d'éviter le gonflement des effectifs permanents. À cela s'ajoute une proportion relativement élevée de "travail au noir ». On peut parler ici d'un système de production, type Sentier, mais sans la proximité immédiate du marché. Donc, malgré la relative rigidité du système en raison de l'intégration verticale de la production, il y a une flexibilité grâce au fonctionnement de la sous-traitance.

Ce système fonctionne parfaitement pour le fabricant qui trouve dans les ressources locales toute la souplesse nécessaire à une industrie ayant des rythmes saisonniers. La flexibilité d'ensemble de l'organisation productive repose sur des relations de confiance, aussi bien entre fabricants et façonniers qu'à l'intérieur de l'entreprise entre le patron et ses ouvriers. Les chefs d'entreprise sont tous originaires de la région. Tout le monde se connaît et a fréquenté les mêmes écoles. On est dans un système de "relations domestiques " (Eymard-Duvernay, 1990). Il règne à Roanne une mentalité traditionnelle très particulière : Roanne ville textile «propre» qui s'oppose à la ville voisine de St-Étienne «ville noire " avec ses mines. On craint les innovations outrancières dans le domaine vestimentaire, on recherche par contre le confort et ce qu'on pourrait définir comme un style " comme il faut ", " impeccable ». L'image de marque de l'industrie roannaise est associée à celle de la femme active ${ }^{14}$, d'âge moyen, utilisant des vêtements pratiques et confortables.

Le tricot permet de réaliser des tissus très souples (jersey) qui ne se froissent pas. La qualité du produit dépend de la matière première utilisée (laine pure pour le haut de gamme, fibres mélangées pour la qualité courante) et du

13. Alors que les entreprises de tissage se sont installées à la périphérie, la maille s'est développée à l'intérieur de la ville. Certains quartiers comme le faubourg Mulsant sont composés presque exclusivement d'entreprises de la maille.

14. À la même époque, près d'une femme sur deux est active et travaille dans la bonneterie/confection. Les ménages disposent d'un double salaire. Roanne est une ville prospère et l'image du produit est en accord avec cette prospérité. 
degré de finition. Elle renvoie tout autant à la qualité des équipements (qualité industrielle) qu'au savoir-faire de la main-d'œuvre utilisée (qualité artisanale).

Un autre aspect du modèle de production traditionnel touche à la dynamique ascensionnelle qui caractérise le textile roannais jusqu'au début des années 1980. Ce mouvement prend sa source dans l'aspiration qu'a tout façonnier à devenir un jour fabricant. On passe ainsi d'un statut social inférieur d'entreprise dominée à un statut supérieur d'entreprise qui intègre la conception des produits et leur commercialisation. Les investissements de base étant peu élevés (quelques métiers à tricoter) l'entreprise débute sous forme artisanale et se compose des membres de la famille du chef d'entreprise, celui-ci étant le plus souvent issu d'une entreprise concurrente. L'entreprise se développe en augmentant progressivement le nombre de machines. L'objectif, à terme, est d'arriver à présenter une collection pour pouvoir échapper à la tutelle du fabricant. Cette collection est généralement élaborée par la femme du chef d'entreprise, capable, mieux qu'une styliste extérieure, de concevoir des modèles adaptés aux performances du matériel en place. On est dans un système qui s'apparente au système du district italien: multitude de petites entreprises, très fortes relations interindustrielles à l'intérieur de la zone, mobilité ascensionnelle qui correspond au passage du statut de façonnier à celui de fabricant, élément moteur du développement régional qu'on peut assimiler à une sorte de voie royale vers la réussite. Ici le modèle de réussite est visible, comme en témoigne une réflexion que nous a faite l'une de nos interlocutrices, responsable d'une petite entreprise de confection : « [...] Ce qui m'a décidé à créer mon entreprise, c'est d'avoir vu M. Désarbre circuler dans une belle voiture rouge, quand j'étais petite... Moi aussi je voulais avoir une belle voiture rouge [...]».

Comme dans le district italien, il existe un savoir-faire régional. Ce savoir-faire renvoie à la notion de tradition et d'héritage, tradition du travail bien fait, transmise de génération en génération. Ce savoir-faire repose sur deux éléments particuliers : le savoir-faire entrepreneurial et le savoir-faire des femmes.

Il existe un « savoir-faire entrepreneurial » issu de la densité des petites et moyennes entreprises qui constituent le tissu industriel roannais. Ce savoir-faire crée une sorte de levier qui pousse à la création d'entreprises, malgré les difficultés conjoncturelles. Il s'associe à un savoir-faire d'entreprises, à une traditionmaison transmise par héritage. Le père était un homme de " métier » connaissant tout de la technique de la maille et il a transmis son savoir-faire à ses enfants. Ce savoir-faire est protégé par le secret, d'autant plus que beaucoup d'entreprises fabriquent le même style de produit. Aujourd'hui, ce savoir-faire n'est plus strictement technique et touche au domaine de la création et de la vente.

On peut dire que la flexibilité du système productif repose en grande partie sur la gestion de l'emploi féminin. Il y a une étroite répartition des tâches 
entre les hommes et les femmes. Les hommes travaillent dans la métallurgie ou conduisent les métiers à tricoter dont ils assurent le réglage et l'entretien, ce qui exige maintenant de plus en plus de formation technique. Les femmes travaillent dans les ateliers de confection, mais leur formation professionnelle reste assez faible. On a affaire ici à deux savoir-faire qui ne se situent pas au même niveau. Chez les femmes, il s'agit d'un savoir-faire non reconnu dans les classifications "proche de leurs tâches ménagères " (coupe, couture, repassage...), alors que chez les hommes, il s'agit d'un savoir-faire professionnel reconnu.

Bien que le système traditionnel roannais se rapproche du district industriel italien, il n'y a pas d'éclatement de la production en une multitude de "phases" qui peuvent s'associer ou rester indépendantes. Le modèle de référence est l' entreprise intégrée tricotage-confection, autour de laquelle gravitent des petits façonniers qui aspirent à devenir eux-mêmes des fabricants. Il n'y a pas à proprement parler de mobilité, sinon à travers le changement de statut entre sous-traitant et fabricant. La prospérité de ce système industriel est liée à la période de croissance économique, et les entreprises se développent en étroite relation avec la croissance des marchés. La logique de développement est une logique de production: les entreprises fabriquent selon un rythme saisonnier des produits à destination du marché intérieur par le système de la distribution de détail. Les entreprises roannaises, à la différence des entreprises italiennes à la même époque sont peu tournées vers l'exportation.

\subsection{L'éclatement du modèle de production traditionnel}

Avec la logique de marché qui est apparue après la crise de 1978/1982, le système traditionnel roannais a été fortement ébranlé. D'autres logiques de modernisation sont apparues. Les conditions de travail se sont beaucoup détériorées, et le consensus qui liait les ouvrières à l'entreprise textile pourvoyeuse d'emplois n'existe plus : les entreprises leader de la région ont beaucoup licencié, certaines ont déposé leur bilan. La sécurité d'emploi qu'offrait cette industrie a disparu, et son "attractivité " a diminué du même coup ${ }^{15}$.

Lors de notre enquête, beaucoup de nos interlocuteurs nous ont parlé du basculement brutal d'une économie de production vers une économie de marché, en situant ce retournement à la fin des années 1970, c'est-à-dire au moment où la crise a touché l'industrie régionale. Jusque-là, le système industriel

15. Il n'y a pas de convention collective locale textile, alors qu'il en existe pour les industries mécaniques. Les ouvrières à domicile ne reçoivent aucune indemnité compensatoire, en cas de baisse d'activité de leur entreprise. Elles sont payées à la pièce, et si elles réclamaient, elles seraient licenciées. Aujourd'hui, il existe une grave pénurie de main-d'œuvre féminine, les femmes préferent travailler dans d'autres secteurs. 
roannais fonctionnait selon un modèle de développement endogène, avec de forts échanges à l'intérieur de la zone, mais peu d'ouvertures à l'extérieur. À partir de cette époque, c'est l'organisation même du système industriel qui est remise en cause et complètement bouleversée. Plusieurs phénomènes se conjuguent pour accélérer la transformation.

Une forte concurrence en provenance d'Italie et des pays à bas coût de main-d'œuvre se développe. La grande distribution pour laquelle travaillent plusieurs producteurs roannais fait pression sur les prix de vente en important de plus en plus massivement des produits bas de gamme.

Les goûts des consommateurs évoluent, en particulier ceux des jeunes, issu du baby-boom, qui disposent d'argent pour acheter des vêtements et refusent de s'habiller comme leurs aînés. Ils veulent se singulariser en portant des vêtements originaux. La " solidité », qualité primordiale aux yeux de la génération précédente, laisse place à la " nouveauté " perpétuellement renouvelée. La volonté de porter un vêtement «décontracté » permet la vente de produits mal finis, les "prêts à jeter » pour reprendre l'expression de Solange Montagné Villette (1990).

La distribution, sous toutes ses nouvelles formes, tend à s'approprier la fonction commerciale et à piloter indirectement la production. Le point de départ de cette évolution résulte d'une saturation du marché et de la nécessité de relancer la demande par un renouvellement permanent des produits proposés : le rythme saisonnier des collections est remis en cause. Les distributeurs sont les mieux placés, étant donné leur proximité du marché, pour susciter de nouveaux besoins et s'y ajuster. Afin de réduire les risques d'invendus, tout en disposant au jour le jour des produits qui se vendent, ils commandent de plus en plus tard, en quantité de plus en plus réduite.

Désormais, les fabricants qui veulent se maintenir doivent tout à la fois opérer une montée en gamme, diversifier leurs produits, en particulier grâce à l'introduction de produits à renouvellement rapide, et réduire leur temps de production. Tout ceci implique un renouvellement des politiques commerciales et une modernisation poussée des équipements. La crise qui secoue la région est un révélateur de l'inadéquation du système industriel local aux transformations du marché. Fabriquant sur cycles longs, des produits de moyenne gamme, peu créatifs, traditionnels, les entreprises ne peuvent plus écouler leurs produits. La conséquence est une réduction importante de l'emploi. Entre 1975 et 1985, les effectifs employés dans la bonneterie baissent de $38 \%$. Cette chute touche principalement les entreprises de plus de 500 personnes dont plusieurs ferment

16. Deux d'entre elles étaient des leaders régionaux spécialisés dans le vêtement d'enfant. 
leurs portes ${ }^{16}$. Les autres stabilisent progressivement leurs effectifs autour de 250 à 300 personnes maximum. Les petites et moyennes entreprises se maintiennent généralement bien. Dans les entreprises de tissage, on enregistre quelques licenciements.

Le Plan textile 1981/1982 ${ }^{17}$ a joué un rôle déterminant dans le maintien en vie du système textile roannais. Il a été d'autant plus bénéfique qu'il coïncidait avec une révolution technologique importante : l'arrivée sur le marché des métiers japonais à commande numérique ${ }^{18}$. La modernisation a touché d'abord les petites entreprises sous-traitantes, contraintes de s'endetter, pour faire face à la pression de leurs donneurs d'ordre. Puis les grandes entreprises se sont équipées et ont récupéré une grande partie de la production précédemment externalisée. Ce Plan a eu des effets pervers sur l'équilibre de l'emploi dans les années suivantes. Les petites entreprises sous-traitantes qui avaient embauché pour faire face à l'accroissement des commandes liées à la modernisation, se sont trouvées en difficulté lorsque les donneurs d'ordre ont diminué leurs commandes, et ont été amenées à diversifier hors région leurs prises de commandes, faute de quoi elles disparaissaient. Les grandes entreprises, tout en récupérant le tricot, ont délocalisé hors de la région le gros des opérations de confection, très utilisatrices de main-d'œuvre féminine locale.

\subsection{Les nouvelles logiques de développement}

À partir de l'enquête que nous avons menée, nous constatons que les changements organisationnels qui ont touché le textile roannais depuis la crise ne débouchent plus sur un modèle d'organisation unique, tel qu'il apparaissait dans la période précédente. Plusieurs logiques de développement se manifestent qui ressortissent à la fois des transformations qui affectent l'ensemble du secteur textile/habillement, et des particularités héritées du passé.

Dans le modèle de production traditionnel, il existait une cohérence entre l'organisation de la production, la qualité du produit, le savoir-faire local. Les prix des produits étaient établis selon le temps de fabrication et la quantité produite, et leur qualité dépendaient de la matière première utilisée, des investissements techniques, du savoir-faire. L'appellation de système industriel localisé prenait tout son sens. Actuellement, la dynamique de développement n'est plus une dynamique endogène, comme celle qui poussait le sous-traitant à devenir fabricant. Elle prend sa source dans la capacité de renouvellement et

17. Ce Plan prévoyait une exonération des charges sociales pendant deux ans pour les entreprises qui modernisaient leurs équipements.

18. Ces métiers permettent de flexibiliser la production : gain de $50 \%$ sur le temps de tricotage, gain de temps pour les changements de modèle. 
d'innovation dont font preuve certaines entreprises. On la voit apparaître en particulier dans le fort mouvement de création/reprises d'entreprises qui s'inscrit dans une dynamique de rupture par rapport au modèle traditionnel de réussite.

Cette évolution d'ensemble se répercute sur la qualité des produits qui n'est plus liée au choix de la matière première et au degré de finition, mais au raccourcissement des délais de production et à la capacité de renouvellement des produits. Les prix sont calculés en fonction des prix des produits importés des pays à bas coût de main-d'œuvre, et non en fonction des temps de fabrication et des coûts de la main-d'œuvre locale. La qualité de type "domestique » (produit fabriqué à Roanne, marque attachée au renom d'une famille et à un savoir-faire d'entreprise) cède la place à une qualité de type " marchand " (produit conçu en fonction de la demande du consommateur, marque de distribution, produit qui n'existe plus en tant que tel et qui peut-être fabriqué n'importe où).

La dynamique ascensionnelle, moteur du modèle de production traditionnel, reposait sur une différenciation très forte entre les statuts des fabricants et ceux des sous-traitants et sur les efforts des seconds pour atteindre le statut des premiers. Cette dynamique est rompue à cause des coûts financiers énormes entraînés par la promotion d'une marque. Le clivage façonniers/petits fabricants s'estompe, alors que se creuse le fossé entre les grands fabricants qui se désengagent de la production pour se consacrer à la promotion de leur marque et les petits producteurs (fabricants et façonniers) qui n'ont pas les moyens de développer une politique de marque.

Nous avons distingué deux grands mouvements autour desquels s'organise la modernisation du système textile roannais.

Une premiere orientation s'inscrit dans le prolongement des tendances développées antérieurement, mais avec des mutations importantes en ce qui concerne la répartition des fonctions entre "fabricant » et "façonnier». On y trouve les grands fabricants dont la réussite a servi de moteur au modèle traditionnel. Ils possèdent une marque grand public et certains ont créé leur propre réseau de franchises. Le coût de plus en plus élevé des investissements commerciaux les conduit à réduire progressivement la part des investissements liés à la modernisation des équipements. Ils ne conservent en fabrication que la réalisation des prototypes et l'échantillonnage des collections. Ils délocalisent la majeure partie de leur production, et quittent leur enracinement local ( $c f$. Pôle I dans le schéma).

Cette évolution s'appuie sur une nouvelle répartition des tâches entre le fabricant qui maîtrise le produit et sa diffusion, et le producteur, enraciné localement, qui gère le processus de production et le savoir-faire local. Le fabricant ne produit plus. Cette situation instaure des relations nouvelles entre 
fabricants et sous-traitants : ces derniers ne sont plus de simples fournisseurs d'heures de travail. L'appellation de «façonnier » devient périmée, et laisse la place à la notion de "producteur ». Certains producteurs s'orientent vers la création de plate-formes techniques regroupant des activités de production complémentaires et constituent une organisation souple de petites entreprises capables de répondre rapidement à la diversité de la demande. D'autres, qui possèdent un potentiel technique et une capacité productive importante, mettent leur savoir-faire industriel au service de leurs donneurs d'ordre ( $c f$. Pôle IV dans le schéma).

Tous ces producteurs (qui représentent une part importante des entreprises roannaises) proposent un ensemble de services adaptés aussi bien aux attentes des fabricants qui se désengagent de la production qu'à celle des créateurs à la recherche d'une grande professionnalité, et surtout des grands distributeurs qui bénéficient ainsi d'une prise en charge de la gestion de toutes les étapes de la fabrication et d'un potentiel de production flexible.

Avec cette première voie, on s'oriente vers un clivage de plus en plus marqué entre des entreprises à vocation commerciale et dont la dynamique de développement n'est plus du tout locale, et des entreprises spécialisées en production, enracinées localement, et très utilisatrices du savoir-faire local, mais aussi très dépendantes de donneurs d'ordre extérieurs à la région.

Une deuxieme orientation s'inscrit dans une dynamique de rupture radicale avec le modèle de réussite traditionnel. Il s'agit d'entreprises nouvellement créées ou reprises à cause des solidarités reliées à l'enracinement local. Ce ne sont pas des filiales d'entreprises extérieures. Elles constituent de petites structures commerciales et sous-traitent dans la région toutes les opérations de production. Elles bénéficient de la grande diversité offerte par le parc machine de la région. Elles émergent dans un tissu industriel ayant accumulé un savoir-faire et une mémoire créative qu'elles exploitent pour fabriquer vite des produits qui se vendent bien.

On trouve ici des entreprises dont la créativité est davantage liée au renouvellement du produit qu'à son style. Elles sont branchées immédiatement et en permanence sur la demande finale grâce à la diversité de leurs sources d'informations. Parmi celles-ci, le Sentier joue un rôle important. Elles ne réalisent pas d'investissements commerciaux auprès de leurs distributeurs comme doivent le faire les grands fabricants possesseurs d'une marque. Chaque produit est conçu en fonction de la spécificité du client, et de ce fait, la structure commerciale est très allégée. Ces entreprises bénéficient de toute la souplesse de fonctionnement du système textile roannais (travail à domicile, temps partiel, travail au noir...), et grâce à cette souplesse, elles peuvent proposer des prix compétitifs à la grande distribution ( $c f$. Pôle II dans le schéma). 
En marge de ces deux grands mouvements de modernisation, il existe une voie médiane dans laquelle se maintiennent certaines entreprises issues du modèle traditionnel roannais. Sans connaître l'aboutissement présenté dans le premier mouvement, elles tentent de maintenir leur indépendance de fabricant en s'appuyant sur leur vocation de «producteur au service d'un produit spécifique ». La solution de repli vers la sous-traitance n'est pas retenue, soit parce que les grands donneurs d'ordre ne sont pas intéressés par ce produit trop particulier, soit à cause de la trop grande rigidité du système de production. Cette dynamique conduit à des volumes de production de plus en plus importants qu'il faut écouler ( $c f$. Pôle III dans le schéma).

Ces entreprises sont poussées à s'imposer sur des créneaux particuliers de la distribution de détail. C'est une voie difficile dans laquelle certaines entreprises ont trouvé un équilibre dynamique. Mineure dans la région, cette voie est intéressante dans la mesure où elle témoigne de la détermination de certaines entreprises à maintenir leur indépendance en investissant dans des équipements très performants. Si l'évolution générale de l'activité n'est pas favorable à cette politique, des réussites individuelles sont néanmoins observées. En tout état de cause, ces réussites laissent entendre que les voies de développement d'une région sont diverses et qu'elles ne rompent pas nécessairement avec la tradition.

\section{Conclusion}

Les résultats de l'étude que nous avons menée à Roanne apportent-ils des éléments de réponse à la question que nous posions au début de cet article au sujet de la capacité des systèmes industriels locaux à promouvoir un nouveau mode de développement économique, face à l'échec actuel du système de production de la grande entreprise ? Il nous semble qu'ils apportent deux éléments de réponse : l'un touche au mode d'organisation propre à un système industriel localisé, l'autre concerne la conservation des savoir-faire.

Contrairement à ce que nous pensions au départ, à la lecture des études faites sur les districts italiens, on ne peut pas dire que le système textile roannais présente un mode de développement homogène, spécifique aux PME qui le composent. La spécialisation flexible n'est pas le modèle d'organisation unique : nous avons vu cœxister à l'intérieur de la même entreprise une production en " grandes séries ${ }^{19}$ » et une production flexible, une spécialisation des tâches et de la polyvalence, des produits mode à renouvellement rapide, et des produits à renouvellement saisonnier.

19. Les séries peuvent varier de 50 à plus de 5000 suivant le type d'article.

Revue internationale P.M.E., vol. 4, ${ }^{\circ} 2,1991$ 
Nous avons constaté par ailleurs une certaine diversité dans les stratégies des firmes, comme en témoignent les quatre grandes tendances d'évolution schématisées dans le tableau. Une des raisons de cette diversité tient à la taille des entreprises : les politiques menées par les «grosses » PME (300 personnes environ) ne sont pas les mêmes que celles des petites (moins de 20 personnes) et présentent des points communs avec celles des grands donneurs d'ordre appartenant à d'autres secteurs d'activité. C'est le cas des politiques de délocalisation de la production vers les pays à bas coûts de main-d'œuvre. Ces entreprises produisent en grandes quantités et sont de moins en moins implantées localement. La dynamique de développement de la région est largement infléchie par cette externalisation d'une part importante de la fabrication régionale. On peut citer ici l'exemple d'une de ces entreprises qui emploie 200 personnes à l'intérieur de ses locaux roannais et dont le chiffre d'affaires correspond en fait à l'emploi hors région de 700 personnes. Quelle différence y a-t-il entre la politique de cette PME et celle d'une grande entreprise ?

Néanmoins, il semble qu'une certaine forme de délocalisation ait atteint ses limites, et que la force du système textile roannais réside dans sa capacité de fabriquer industriellement (c'est-à-dire en quantité) aussi bien des produits hauts de gamme, que des produits créatifs à renouvellement rapide. Grâce à un parc machine très diversifié et dispersé dans des petites entreprises, dans lesquelles voisinent des équipements anciens avec des équipements modernes adaptés chacun à des produits spécifiques, on peut en effet penser que ce système est capable de s'adapter aux nouvelles exigences du marché mondial. Mais là encore, qu'y a-t-il de spécifique par rapport aux grandes entreprises automobiles qui après des tentatives de mondialisation de leurs stratégies industrielles retrouvent, dans le regroupement de leurs équipementiers dans une même zone géographique, les avantages financiers liés à la production en " juste à temps? » (De Banville et Chanaron, 1991)

Autour des grands donneurs d'ordre roannais, gravitent une quantité de petites entreprises qui fonctionnent de manière extrêmement souple, grâce à la flexibilité du travail féminin (travail à domicile, temps partiel, chômage partiel non déclaré, travail au noir). Elles possèdent généralement un équipement moderne ce qui est un atout majeur face aux contraintes imposées par la diversification des séries et par l'accélération des rythmes de production. Elles sont en quelque sorte un réservoir de travail flexible. Et là, on retrouve la flexibilité à l'italienne avec une connotation accentuée de flexibilité technique.

Cette nébuleuse de petites entreprises est organisée à la fois autour des entreprises locales importantes et autour de donneurs d'ordre hors région. Leur organisation productive privilégie le raccourcissement des circuits de production et le renouvellement accéléré des produits. La force des entreprises apparues depuis la crise a été de s'appuyer sur la souplesse de ce système de production 
traditionnel. Mais il ne faut pas oublier que la souplesse de la production repose sur l'exploitation du travail des femmes et sur leur adhésion à ces formes de travail flexible. Or, les jeunes femmes ont tendance à quitter le textile et à s'orienter vers des métiers mieux rémunérés et réclament une formation professionnelle. On peut se poser la question de l'avenir du système textile roannais si les femmes désertent l'industrie textile.

Un autre trait spécifique aux systèmes industriels locaux, particulièrement visible dans la région roannaise, concerne la conservation des savoir-faire locaux. Les PME sont en quelque sorte les gardiennes de ce savoir-faire face à la politique de «terre brûlée » menées par les grands groupes textile.

Nous avons constaté à Roanne que certains chefs d'entreprise jouent un rôle déterminant dans la valorisation de ce savoir-faire ${ }^{20}$. Comme on l'a vu précédemment, ce savoir-faire est aussi bien un savoir-faire entrepreneurial qui pousse les individus à créer leur propre entreprise plutôt qu'à travailler comme salarié, qu'un savoir-faire lié à une longue expérience des métiers du textile. Le statut de chef d'entreprise reste encore un statut attractif dans cette région de PME qui comporte un grand nombre de chefs d'entreprises.

On peut signaler enfin que les chefs d'entreprises de PME sont des « employeurs » et qu'ils se sentent responsables du maintien de l'emploi de leur personnel, même si l'entreprise traverse des difficultés. Cette attitude, qui s'enracine dans une vieille tradition régionale, joue un rôle important dans le maintien global de l'emploi dans la région.

Cette capacité de constituer une sorte d'amortisseur souple entre les exigences de la production et celles du marché, grâce à leur organisation flexible et à leur utilisation du savoir-faire régional nous paraît être le meilleur atout du système textile roannais à l'heure actuelle. Mais qu'en resterait-il si les grands leaders locaux quittaient la région pour des raisons liées à leurs nouvelles stratégies commerciales ? C'est là, nous semble-t-il, que l'on touche les limites des systèmes industriels localisés.

20. Un industriel du tissage, qui est une des personnalités les plus marquantes de la région, est à l'origine de la reprise de différentes entreprises textiles, dont une très importante de tissage qui appartenait primitivement au groupe DMC. 


\section{Bibliographie}

Bagnasco, A. (1987), Tre Italia La problematica territoriale dello sviluppo italiano, Bologna Editions II Mulino.

De Banville, E. et J.J. Chanaron (1991), Vers un système automobile européen, Paris, Economica.

Beccatin, G. et alii (1987), Mercato e forze locali : il distretto industriale, Editions Il Mulino.

Brusco, S. (1980), «Il modello Emilia: desintegrazione produttiva e integrazione sociale », Communication à l'Institut GRAMSCI. Traduction in Cambridge Journal of Economics (1982) $n^{\circ} 6$.

Courault, B. et F. Rérat (1987), «Un modèle de production régionale en transition : le cas de la chaussure dans le choletais », Paris, Cahiers du CEE, $\mathrm{n}^{\circ} 30$, PUF.

Courault, B. et F. RÉRAT (1988), «La flexibilité de la production », dans Les stratégies d'entreprises face aux ressources humaines, L'après-Taylorisme, Paris, F. Stankiewicz éditeur, Economica.

Courault, B., F. Rérat et R. Weisz (1989), «Footwear manufucturing firms in France : a typological study ", Labour and Society, Genève, B.I.T., avril.

Courault, B., F. Rérat, F. Rubéry, G. Solinas, P. Vila, R. Weisz et F. Wilkinson (1990), «L'industrie de la chaussure en Europe : vers plus de flexibilité », Dossier de recherche $n^{\circ} 35$ du Centre d'Études de l'Emploi, Noisy le Grand.

EyMARD-DuVERnAY, F. et O. FAVEREAU (1990), « Marchés internes, modèles d'entreprises et conventions de qualités », Communication aux $7 e$ journées de micro-économie appliquée, Université du Québec à Monréal.

Eymard-Duvernay, F. (1990), "Conventions de qualité et formes de coordination », Revue Économique, Paris.

Ganne, Saglio, Baptiste, Micheison et McArthur (1988), «Milieux industriels et systèmes industriels locaux, une comparaison France-Italie », GLYSI, CNRS, Lyon, janvier.

GANNE, B. (1989), «Industrialisation diffuse et systèmes industriels localisés », Essai de bibliographie critique du cas français. GLYSI - MRSH, mars, $\mathrm{n}^{\circ} 2$, Rapport pour l' IIES - BIT Genève. Financé par la «TSAK Force PME » de la Commission des Communautés européennes.

Houssel, J.P. (1978), Le Roannais et le Haut Beaujolais : un espace à l'écart des métropoles, Lyon, Presses Universitaires de Lyon.

LAFÈRÈRE, M. (1960), Lyon Ville industrielle, Paris, PUF.

LORTHIOIS, J. et F. RÉRAT (1987), « Ouvrières : savoir-faire en sursis ? », Pour, Éd. Privat, Toulouse, $\mathrm{n}^{\circ} 108$.

Minguet, G. (1985), La naissance de l'Anjou industrielle, Paris, Éditions l'harmatan, Collection Logiques Sociales.

Montagné Villette, S. (1990), Le Sentier, un espace ambigu, Paris, Masson.

PIORE, M. et C. SABEL (1984), The Second Industrial Divide, New York, Basic Books Inc.

RÉRAT, F. (1986), «La polyvalence comme méthode d'organisation du travail » dans Formation Emploi, Doc. Fse. $\mathrm{n}^{\circ} 14$, avril-juin. 
RÉRAT, F., N. Bescuso et B. Gomel (1990), «Le textile à Roanne. Logiques de développement d'un système industriel localisé », Dossier de Recherche, $\mathrm{n}^{\circ} 36, C . E . E$, Paris.

RoveYRE, M.F. et J. SAGLIo (1984), «Les systèmes industriels localisés : éléments pour une analyse sociologique des ensembles de PME industriels », Sociologie du Travail, $\mathrm{n}^{\circ} 2$, Paris.

SaGlio, J. (1987), "Préléminaires pour une typologie des systèmes industriels », Communication à un séminaire du Gréco relations industrielles, Paris, CNAM, mars.

SolinAs, G. (1982), «Labour market segmentation and workers career : the case of the italian knitwear industry », Cambridge Journal of Economics, $\mathrm{n}^{\circ} 6$. 